\title{
Factors affecting interictal unilateral and bilateral discharges and ictal diffusion patterns of scalp electroencephalogram in temporal lobe epilepsy
}

\author{
Weining Ma ( $\sim$ maweining1985@163.com ) \\ Shengjing Hospital of China Medical University \\ Han Li \\ Shengjing Hospital of China Medical University

\section{Pengyu Chen} \\ Shengjing Hospital of China Medical University \\ Miaomiao Li \\ Shengjing Hospital of China Medical University \\ Liping Liu \\ Shengjing Hospital of China Medical University \\ Lin Cong \\ Shengjing Hospital of China Medical University \\ Chun Li \\ Shengjing Hospital of China Medical University \\ Shaoyi Li \\ Shengjing Hospital of China Medical University
}

\section{Research article}

Keywords: Temporal lobe epilepsy; Interictal discharge; Ictal electroencephalogram

Posted Date: August 22nd, 2019

DOl: https://doi.org/10.21203/rs.2.13397/v1

License: (c) (i) This work is licensed under a Creative Commons Attribution 4.0 International License. Read Full License

Version of Record: A version of this preprint was published at Neurological Sciences on May 4th, 2021. See the published version at https://doi.org/10.1007/s10072-021-05293-0. 


\section{Abstract}

Purpose To evaluate preoperative factors influencing unilateral and bilateral interictal discharges in TLE, and ictal diffusion patterns in scalp EEG during onset of the seizure.Methods This was a retrospective analysis of 129 patients with TLE who had undergone surgery; cases were classified into two groups of unilateral and bilateral discharge groups according to interictal discharge patterns in the EEG. In this study, unilateral interictal discharges meaned that the discharge was confined to one hemisphere, while bilateral interictal discharges meaned that the discharge involved both hemispheres. Differences between the two groups in age, gender, duration of disease, frequency of seizures, MRI findings, origin of TLE, antiepileptic drug administration, and ictal diffusion patterns during seizures were statistically analyzed. In addition, the differences in ictal diffusion patterns between left and right TLE were statistically analyzed.Results There were a total of 74 cases of unilateral discharges, including unilateral discharges in the temporal region, anterior head including both temporal and frontal regions, and unilateral hemispheric discharges, and there were another 55 cases of bilateral discharges, including bilateral temporal area, bilateral anterior heads, and bilateral hemispheric multi-brain areas. There were 60 cases of left TLE and 69 cases of right TLE. There were no statistically significant differences in gender, duration of disease, frequency of seizures, MRI manifestations, administration of antiepileptic drugs, and ictal diffusion patterns between interictal unilateral and bilateral discharge groups, but, there were statistically significant differences in age and side of origin of the TLE. Scalp EEG recorded 11 different ictal diffusion pattern types, and the difference in terms of whether the EEG recorded the diffusion pattern to be confined to the same hemisphere or spread to both hemispheres was statistically significant between the left and right temporal lobes.Conclusion Age and side of origin of TLE affects the TLE interictal discharge patterns. Older patients are more prone to bilateral discharges. Bilateral discharges are more common in right TLE, and the onset of EEG more likely to bilateral diffusion in right TLE.

\section{Background}

Epilepsy is a common neurological disease, and temporal lobe epilepsy (TLE) is the most common type of drug-resistant epilepsy (DRE) (1), accounting for about $70 \%$ of cases of epilepsy (2). Electroencephalogram (EEG) is an important examination method for epilepsy diagnosis and treatment planning. In epilepsy surgery, the determination and localization of epileptic foci are the core issues in the operative planning, and EEG examination is an important basis for making surgical resection plan. The location and range of interictal discharge, such as unilateral or bilateral interictal discharges, have important guiding significance for the determination and localization of epileptic foci, as well as the prognosis of surgery. Besides, the ictal EEG is used to locate the origin of the seizure. One study (3) has shown that bilateral interictal discharges before operation indicated poor prognosis of epilepsy surgery. Therefore, it is very important to predict the prognosis of the operation by means of the range of involvement of interictal EEG in order to reduce the risk of operation and possible complications. Surgery is an effective way to treat TLE (4) and EEG is an important method of preoperative assessment of this seizure disorder. However, TLE is often associated with neuropsychological impairment (5), which may be related to the affected range of interictal EEG in TLE (6). Clinically, unilateral or bilateral (synchronous or asynchronous) discharge patterns in interictal EEG in TLE are very common in clinical practice. Furthermore, the diffusion range of EEG in the ictal phase is often significantly larger than that in the interictal phase. Therefore, it is very important to evaluate the degree of neurological impairment and to predict the prognosis of surgery through interictal and ictal discharge patterns of TLE. What, then, are the factors associated with the different discharge patterns of TLE, one might ask? Does duration of the disease and frequency of seizures lead to an increase in the discharge range? Is the pattern of ictal diffusion during a seizure related to the pattern of interictal discharge? Are there differences in ictal diffusion patterns between the left and right temporal lobes during seizures? Therefore, based on the above issues, this study retrospectively analyzed 129 patients with TLE who had undergone surgery, along with analysis of preoperative interictal and ictal EEG data. The differences in age, gender, duration of disease, seizures frequency, magnetic resonance imaging (MRI) performance, side of origin of TLE, antiepileptic drug (AED) administration, and ictal diffusion patterns between unilateral and bilateral discharge groups were statistically analyzed. In addition, the differences in ictal diffusion patterns between left and right TLE were also statistically analyzed.

\section{Methods}

Clinical case collection

Page 2/14 
A total of 129 cases of TLE who had undergone surgery were retrieved from Shengjing Hospital affiliated to China Medical University, Functional Neurosurgery Unit. Data related to age, gender, duration of disease, seizure frequency, types of antiepileptic drugs, interictal and ictal EEG, and MRI performance were collected. The study was approved by the Ethics Committee of China Medical University to which Shengjing Hospital is affiliated and patients provided written, informed consent for the use of their data.

\section{Classification of interictal discharge patterns}

Unilateral discharge patterns include: unilateral temporal area discharges, unilateral anterior head discharges (Figure $1 \mathrm{~A})$, and unilateral hemispheric discharge (Figure 1B). Bilateral discharge patterns include: bilateral temporal area discharges, bilateral anterior head discharges, and bilateral hemispheric multi-brain area discharges. Bilateral synchronous (Figure 1C) or asynchronous (Figure 1D) discharge patterns are counted as bilateral discharges.

\section{Classification of ictal diffusion patterns}

Labeling was carried out according to the order of site of origin and direction of diffusion. The study stipulated no more than three steps of labeling the diffusion process. The onset of temporal and frontal areas were labeled as anterior head.

\section{Statistical analysis}

The t-test was used to analyze statistical differences in age, gender, duration of disease, and seizure frequency between the unilateral and bilateral discharge groups. The Chi-squared test for independence was used to compare differences in antiepileptic drug administration, MRI performance, side of origin of epilepsy, and ictal diffusion patterns between the above two groups and also left and right TLE. $P<0.05$ was considered statistically significant.

\section{Results}

\section{Characteristics of the study population}

The study population consisted of 129 patients, including 66 (51.2\%) males and 63 (48.8\%) females ranging in age from 2 to 62 years with a mean age of $32 \pm 12$ years. The duration of disease was from 0.1 year to 40 years with an average duration of 12.4 years. The seizure frequency was from 0.5 time/month to 45 times/month with an average frequency 6.6 times/month. There were 60 (46.5\%) left TLE cases, and 69 (53.5\%) right TLE cases. There were 44 (34.1\%) cases with normal MRI results and 85 $(65.9 \%)$ cases with distinct abnormalities on MRI. AEDs included levetiracetam (LEV), ocazepine (OXC), clonazepam (CZP), valproate (VPA), lamotrigine (LTG), phenytoin (PHT), phenobarbital (PB), carbamazepine (CBZ), topiramate (TPM), and gabapentin (GBP) (Table 1).

\section{Statistical results of factors affecting the interictal discharge patterns}

The age of the unilateral discharge group ranged from 2 years to 50 years, with an average age of 29 years, while the age of the bilateral discharge group ranged from 7 years to 62 years, with an average age of 35 years. The difference in age between the two groups was statistically significant $(P=0.02<0.05)$ (Figure 2A). The duration of disease in the unilateral discharge group ranged from 0.1 to 35 years, with an average duration of 11.6 years. The duration of disease in the bilateral discharge group ranged from 0.1 to 40 years, with an average course of 13.5 years. There was no statistically significant difference in the duration of disease between the two groups $(P=0.25>0.05)$ (Figure 2B). In the unilateral discharge group, the seizure frequency ranged from 0.5 time/month to 45 times/month, with an average seizure frequency of 6.8 times/month. In the bilateral discharge group, 
the seizure frequency ranged from 1 time/month to 30 times/month, with an average seizure frequency of 6.5 times/month. There was no significant difference in the seizure frequency between the two groups $(P=0.84>0.05)$ (Figure $2 \mathrm{C}$ ). In the unilateral discharge group, there were 21 (28.4\%) cases with no clear MRI abnormalities (negative) and 53 (71.6\%) cases with clear abnormalities (positive), and the numbers were 23 (41.8\%) and 32 (58.2\%), respectively, in the bilateral discharge group. The difference in proportion of MRI with or without abnormalities between the two groups was not statistically significant $(P=0.11>$ 0.05) (Figure 2D). There were $33(44.6 \%)$ cases which originated in the right temporal lobe and 41 (55.4\%) cases which originated in the left temporal lobe in the unilateral discharge group, and 36 (65.5\%) cases originating in the right temporal lobe and 19 $(34.5 \%)$ cases originating in the left temporal lobe in the bilateral discharge group. The difference in proportion of side of origin between the two groups was statistically significant $(P=0.02<0.05)$ (Figure 2E). There were $43(58.1 \%)$ males and $31(41.9 \%)$ females in the unilateral discharge group, and 23 (41.8\%) males and 32 (58.2\%) females in the bilateral discharge group; however, the difference in gender ratio between the two groups was not statistically significant $(P=0.07>0.05)$ (Figure 2F).

AEDs administered in the unilateral discharge group included LEV, OXC, CZP, VPA, LTG, PHT, PB, CBZ, TPM, and GBP, with CBZ, VPA, and PB being the top three utilized drugs (Figure 3A). AEDs administered in the bilateral discharge group included LEV, OXC, CZP, VPA, LTG, PHT, PB, CBZ, and TPM. The top four utilized drugs were CBZ, VPA, PB, and PHT (Figure 3A). In the unilateral discharge group, there were 10 (13.5\%) cases with no AED administration, 39 (52.7\%) cases with single AED administration, and 25 (33.8\%) cases with administration of multi-AEDs (two or more drugs) (Figure 3B). In the bilateral discharge group, there were 6 (10.9\%) cases in whom no AEDs were given, 22 (40.0\%) cases in whom a single AED was given, and 27 (49.1\%) cases with multiAED treatment (Figure $3 \mathrm{C}$ ). There was neither any significant difference in the proportion of cases receiving a single AED vis-a-vis multiple AEDs between the two groups $(P=0.09>0.05$ ) (Figure 3D) nor any significant difference between the two groups in terms of the proportion of cases with no AED treatment vis-a-vis AED treatment $(P=0.66>0.05)$ (Figure 3E).

The above data shows that older patients with TLE were more inclined to discharge bilaterally as seen in the interictal EEG. Moreover, right TLE was more likely to be bilateral as seen in the interictal EEG.

\section{Statistical results of ictal diffusion patterns}

The site of origin of the seizure and the diffusion direction on ictal EEG in the 129 cases were analyzed. They were classified into 11 diffusion patterns, including unilateral anterior head (6.30\%), unilateral anterior head to ipsilateral hemisphere (2.36\%), unilateral anterior head to ipsilateral hemisphere to bilateral hemispheres (6.30\%), unilateral anterior head to bilateral hemispheres (35.43\%), unilateral anterior head to bilateral anterior heads (3.94\%), unilateral anterior head to bilateral anterior heads to bilateral hemispheres $(17.32 \%)$, bilateral anterior heads $(1.57 \%)$, bilateral anterior heads to bilateral hemispheres $(11.81 \%)$, bilateral hemispheres $(12.60 \%)$, unilateral hemisphere $(1.57 \%)$, and unilateral hemisphere to bilateral hemispheres $(0.79 \%$ ) (Figure 6 C). It could be seen that in TLE, the side of origin can be located by scalp EEG most of the time, and the spread of ictal discharge in the EEG in most cases had a wide diffusion range, with most of them having bilateral hemispheric involvement. The cases with interictal unilateral discharge showed all 11 ictal diffusion patterns described above (Figure 6A), while the cases with interictal bilateral discharge showed only 8 patterns (Figure 6A).

When analyzed according to the side of TLE, the ictal diffusion patterns in right TLE included the first nine ictal diffusion patterns mentioned above (Figure 4A through 4G, Figure 6B), and the ictal diffusion patterns in left TLE had all 11 of the patterns described (Figure 5A through 5I, Figure 6B). On statistical analysis, there was no significant difference between the unilateral discharge group and the bilateral discharge group in the ratio of ictal EEG limited to the ipsilateral hemisphere to ictal EEG where the seizure had spread to both hemispheres $(P=0.12>0.05$ ) (Figure 6D). However, there was a statistically significant difference in this ratio between right and left TLE $(P=0.02<0.05)$ (Figure 6E). This demonstrated that right TLE was more likely to spread to bilateral hemispheres during seizures.

\section{Discussion}

The interictal discharge neural network of refractory TLE may be relatively fixed 
In this study, the average age of the unilateral discharge group was older than that of the bilateral discharge group, but there was no significant difference in the length of disease duration between the two groups, that is to say, the average age of disease onset of the unilateral discharge group was older than that of the other group. As far as we know, there is no research on the relationship between onset age and interictal discharge patterns of TLE. Epilepsy may be a kind of neurodegenerative disease. Our results showed that older patients were more prone to bilateral discharge, which may be related to degenerative changes of the nervous system with age, making the neural network more susceptible to generalization. This retrospective study also found that the interictal discharge patterns of TLE were not affected by the patient's gender, duration of disease, frequency of seizures, MRI manifestations, and administration of antiepileptic drugs. Our results showed that there was no correlation between duration of TLE and interictal unilateral or bilateral discharge pattern, suggesting that interictal discharge is not a unilateral to bilateral process of evolution. In addition, there was no clear causal relationship between the range of the interictal brain area affected and frequency of seizures, suggesting that the interictal distribution in TLE may have its own fixed neural network $(7,8)$, and the frequency of seizures may be related to degree of activation of the interictal discharge fixed neural network. Clinically, most patients with TLE exhibit drug resistance, or evolve into drug resistant cases. There are many theories and hypotheses about the causes of drug resistance in TLE (9). There have also been many studies to predict whether TLE can evolve into drug resistance through the effect of initial drug treatment (10) and seizure frequency (11). Furthermore, several studies have speculated about the possible mechanism of drug resistance in TLE by detecting levels of some target proteins, genes, and molecules (12-14). In this study, the average duration of disease was 12.4 years, and the average seizure frequency was 6.6 times/month. These two indicators satisfied the diagnostic criteria for intractable TLE, and, hence, all the selected cases were surgically treated. According to our results, once TLE becomes refractory to drugs, the type of AEDs or whether to choose drug treatment, as well as the decision to administer a single AED or multi-AEDs, did not affect the interictal discharge patterns of TLE, that is, these parameters could not affect the formed neural network of epilepsy (15). Refractoriness to AEDs was evidence of drug resistance, and also indirectly proved the fixed characteristics of the TLE neural network. Therefore, we speculate that the interictal discharge patterns of TLE are determined by its interictal neural network (16). Once formed, it is relatively fixed and not affected by the duration of disease, frequency of seizures, and drug factors.

The difference in the interictal discharge pattern and the ictal distribution between left and right TLE may be based on different neural networks

According to our findings, right TLE accounted for a higher proportion of bilateral discharges. The right TLE was more prone to bilateral discharges than the left TLE in interictal EEG. In addition, right TLE was more inclined to spread to both hemispheres than left TLE, as far as the ictal diffusion patterns in EEG were concerned, while the discharge in left TLE was more commonly limited to the same hemisphere during the seizure, as shown in ictal diffusion patterns in EEG. From the perspective of function, the neural function of the left temporal lobe is different from that of the right temporal lobe. For example, the left temporal lobe is generally closely related to memory and speech, while the right temporal lobe is often closely related to emotion, which also indicates that the two lobes have differences in the normal functional neural network. So, might there be a different epileptic neural network in left and right TLE, resulting in differences in the interictal discharge patterns, the ictal diffusion patterns, and the brain range of involvement during the seizure? Using imaging studies, some workers have proved differences in the neural network and functional anatomy between left and right TLE. A study by Sanjari Moghaddam H. et al. (17) showed that left and right medial TLE (mTLE) had discrepant alternations in the white matter microstructure, including inferior longitudinal fasciculus, forceps minor, genu of corpus callosum, bilateral corticospinal tracts, and bilateral middle cerebellar peduncles, which indicated that left and right mTLE have different underlying pathologic mechanisms. Fang P. et al. (18) found that there were anatomical connectivity differences between left and right mTLE and that left mTLE could be distinguished from right mTLE (19). According to this study, the EEG of right TLE showed that the seizure spread more easily to both sides than the left side in both interictal and ictal periods. We know that the medial temporal lobe structures belong to the limbic system. The loop of the limbic system (Papaz loop) is an important pathway connecting bilateral temporal lobes, and right mTLE often easily damages and affects the limbic system loop (20). This may be a possible factor why right TLE has a wider range of influence than left TLE. Our study also found that in both left and right TLE, the proportion of bilateral hemispheres involved during the seizure, as recorded in the EEG, was very high. Clinically, TLE secondary to generalized seizures is not uncommon, and studies have shown that there are many 
connections between the two temporal lobes (21), and extensive connections with other brain regions, including the frontal lobe, occipital lobe, and thalamus. Furthermore, the occurrence of generalized seizures secondary to TLE is related to the change of hippocampal-thalamic association (22). Therefore, the wide range of EEG findings during the seizure may be related to the extensive neural connections between the temporal lobe and other regions of the brain (23).

\section{Conclusions}

Our study found that age and side of temporal lobe epilepsy affected the interictal discharge patterns of TLE. The elderly are more prone to bilateral discharge, and bilateral discharge is more common in right temporal lobe epilepsy, with ictal EEG more commonly showing bilateral diffusion.

\section{Declarations}

\section{Author contributions}

All authors were involved in the study design, interpretation of the results, and the reviewing and approval of the manuscript, and in the decision to submit the article for publication. All authors also confirm accountability for the accuracy and integrity of the work.

\section{Consent for publication}

All authors consent to the publication of this manuscript.

\section{Ethics approval and consent to participate}

Informed consent from each patient was carried out before the research. The use of the research protocol was approved by the Ethics and the Human Research Review Committee of Shengjing Hospital affiliated to China Medical University.

\section{Funding}

This research did not receive any specific grant from funding agencies in the public, commercial, or not-for-profit sectors.

\section{Acknowledgements}

Not applicable.

\section{Competing interests}

The authors declare that they have no competing interests.

\section{References}

1. Wiebe S. Definition of drug-resistant epilepsy: Is it evidence based? Epilepsia. (2013) 54(Suppl. S2): 9-12.

2. Larsson K, Eeg-Olofsson. A population based study of epilepsy in children from a Swedish county. Eur J paediatr Neurol. (2006) 10(3):107-113. 
3. Shin JH, Joo EY, Seo DW, Shon YM, Hong SB, Hong SC. Prognostic factors determining poor postsurgical outcomes of mesial temporal lobe epilepsy. PLoS One.(2018) 13(10): e0206095.

4. Deleo F, Garbelli R, Milesi G, Gozzo F, Bramerio M, Villani F, Cardinale F, Tringali G, Spreafico R, Tassi L. Short- and longterm surgical outcomes of temporal lobe epilepsy associated with hippocampalsclerosis: Relationships with neuropathology. Epilepsia. (2016) 57(2): 306-315.

5. Pustina D, Doucet G, Skidmore C, Sperling M, Tracy J.

Contralateral interictal spikes are related to tapetum damage in left temporal lobe epilepsy. Epilepsia.(2014) 55(9): 1406-14

6. Dinkelacker V, Xin X, Baulac M, Samson S, Dupont S. Interictal epileptic discharge correlates with global and frontal cognitive dysfunction in temporal lobe epilepsy. Epilepsy Behav.(2016) 62: 197-203.

7. Bonilha L, Nesland T, Martz GU, Joseph JE, Spampinato MV, Edwards JC, Tabesh A. Medial temporal lobe epilepsy is associated with neuronal fibre loss and paradoxical increase in structural connectivity of limbic structures. $J$ Neurol Neurosurg Psychiatry.(2012) 83(9): 903-90

8. Tong X, An D, Xiao F, Lei D, Niu R, Li W, Ren J, Liu W, Tang Y, Zhang L, Zhou B, Gong Q, Zhou D. Realtime effects of interictal spikes on hippocampus and amygdala functional connectivity in unilateral temporal lobe epilepsy: An EEG-fMRI study. Epilepsia.(2019) doi: 10.1111/epi.14646.

9. Tang F, Hartz AMS, Bauer B. Drug-Resistant Epilepsy: Multiple Hypotheses, Few Answers. Front Neurol.(2017) 8 :

10. Bjørke AB, Nome CG, Falk RS, Gjerstad L, Taubøll E, Heuser K. Evaluation of long-term antiepileptic drug use in patients with temporal lobe epilepsy: Assessment of risk factors for drug resistance and polypharmacy. Seizure.(2018) 61: 63-70.

11. Löscher W, Brandt C. High seizure frequency prior to antiepileptic treatment is a predictor of pharmacoresistantepilepsy in a rat model of temporal lobe epilepsy. Epilepsia.(2010) 51(1): 89-97.

12. Banerjee Dixit A, Sharma D, Srivastava A, Banerjee J, Tripathi M, Prakash D, Sarat Chandra P. Upregulation of breast cancer resistance protein and major vault protein in drug resistantepilepsy. Seizure.(2017) 47: 9-12.

13. Ghosh C, Hossain M,Solanki J, Najm IM, Marchi N, Janigro D. Overexpression of pregnane X and glucocorticoid receptors and the regulation of cytochrome P450 in human epileptic brain endothelial cells. Epilepsia.(2017) 58(4): 576-585.

14. Silva-Alves MS, Secolin R, Carvalho BS, Yasuda CL, Bilevicius E, Alvim MK, Santos RO, Maurer-Morelli CV, Cendes F, LopesCendes I. A prediction algorithm for drug response in patients with mesial temporal lobe epilepsy basedon clinical and genetic i PLoS One.(2017) 12(1): e0169214.

15. Karunakaran S, Rollo MJ, Kim K, Johnson JA, Kalamangalam GP, Aazhang B, Tandon N. The interictal mesial temporal lobe epilepsy network. Epilepsia.(2018) 59(1): 244-258.

16. Wang H, Huang Y, Coman D, Munbodh R, Dhaher R, Zaveri HP, Hyder F, Eid T.

Network evolution in mesial temporal lobe epilepsy revealed by diffusion tensor imaging. Epilepsia.(2017) 58(5): 824-834.

17. Sanjari Moghaddam H, Rahmani F, Aarabi MH, Nazem-Zadeh MR, Davoodi-Bojd E, Soltanian-Zadeh H. White matter microstructural differences between right and left mesial temporal lobe epilepsy. Acta Neurol Belg.(2019) doi: 10.1007/s13760-019-01074-x.

18. Fang P, An J, Zeng LL, Shen H, Chen F, Wang W, Qiu S, Hu D. Multivariate pattern analysis reveals anatomical connectivity differences between the left and right mesial temporal lobe epilepsy. Neuroimage Clin.(2015) 7: 555-5

19. Chiang S, Stern JM, Engel J Jr, Levin HS, Haneef Z. Differences in graph theory functional connectivity in left and right temporal lobe epilepsy. Epilepsy Res.(2014) 108(10):1770-17

20. Zhao F, Kang H, You L, Rastogi P, Venkatesh D, Chandra M. Neuropsychological deficits in temporal lobe epilepsy: A comprehensive review. Ann Indian Acad Neurol.(2014) 17(4): 374-3

21. Lacuey N, Zonjy B, Kahriman ES, Kaffashi F, Miller J, Lüders HO.

Functional connectivity between right and left mesial temporal structures. Brain Struct Funct.(2015) 220(5): 2617-26

22. Chen C, Li H, Ding F, Yang L, Huang P, Wang S, Jin B, Xu C, Wang Y, Ding M, Chen Z, Wang S. Alterations in the hippocampalthalamic pathway underlying secondarily generalized tonic-clonic seizures in mesial temporal lobe epilepsy: A diffusion tensor imaging study. Epilepsia.(2019) 60(1): 121-130.

Page $7 / 14$ 
23. de Campos BM, Coan AC, Lin Yasuda C, Casseb RF, Cendes F. Largescale brain networks are distinctly affected in right and left mesial temporal lobe epilepsy. Hum Brain Mapp.(2016) 37(9): 3137-31

\section{Tables}

Table 1

\begin{tabular}{|c|c|c|}
\hline \multicolumn{3}{|c|}{ The demographic of the cases } \\
\hline \multicolumn{2}{|c|}{ The total number of cases } & 129 \\
\hline \multicolumn{2}{|c|}{ Age (year) } & 2-62 (AVE $32 \pm 12$ ) \\
\hline \multirow[t]{2}{*}{ Sex } & Male & 66 \\
\hline & Female & 63 \\
\hline Origin of Seizure & Left & 60 \\
\hline (TLE) & Right & 69 \\
\hline \multirow[t]{2}{*}{ MRI } & Negative & 44 \\
\hline & Positive & 85 \\
\hline \multirow[t]{2}{*}{ Interictal EEG } & Unilateral discharge & 74 \\
\hline & Bilateral discharge & 55 \\
\hline \multicolumn{2}{|c|}{ Duration of disease (year) } & 0.1-40 (AVE 12.4) \\
\hline \multicolumn{2}{|c|}{ Seizure frequency (times/month) } & 0.5-45 (AVE 6.6) \\
\hline \multirow{2}{*}{\multicolumn{2}{|c|}{ AEDs }} & LEV, OXC, CZP, VPA, LTG, \\
\hline & & PHT, PB, CBZ, TPM, GBP \\
\hline
\end{tabular}

\section{Figures}


A

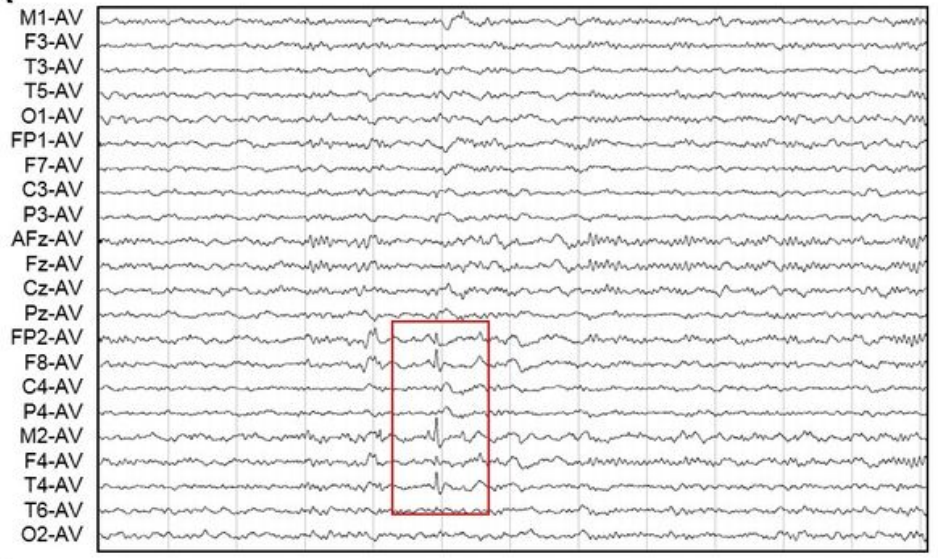

C

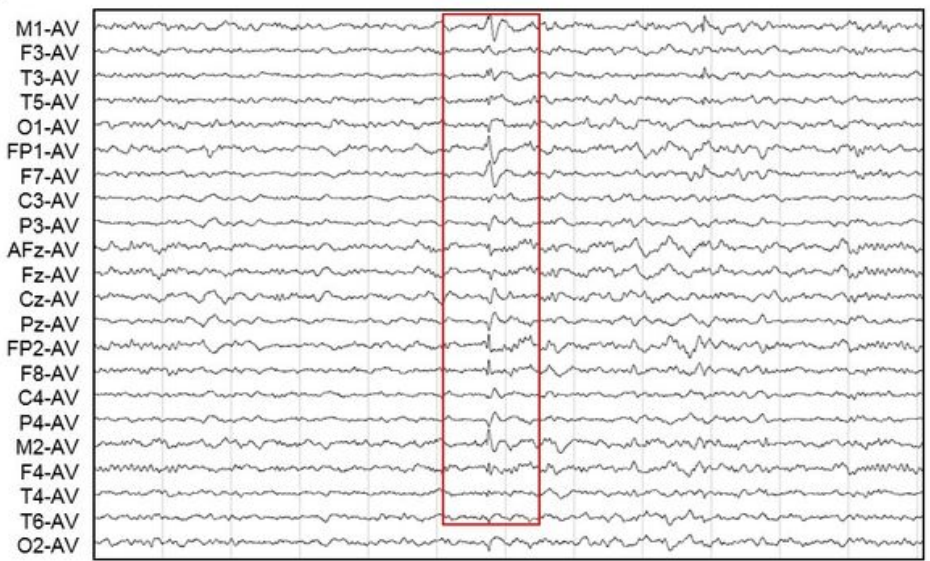

B

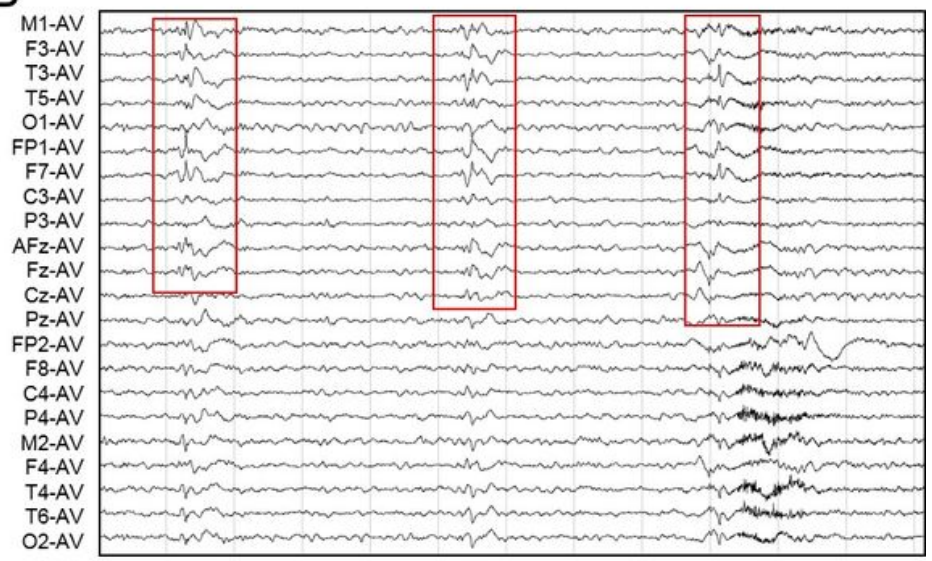

D

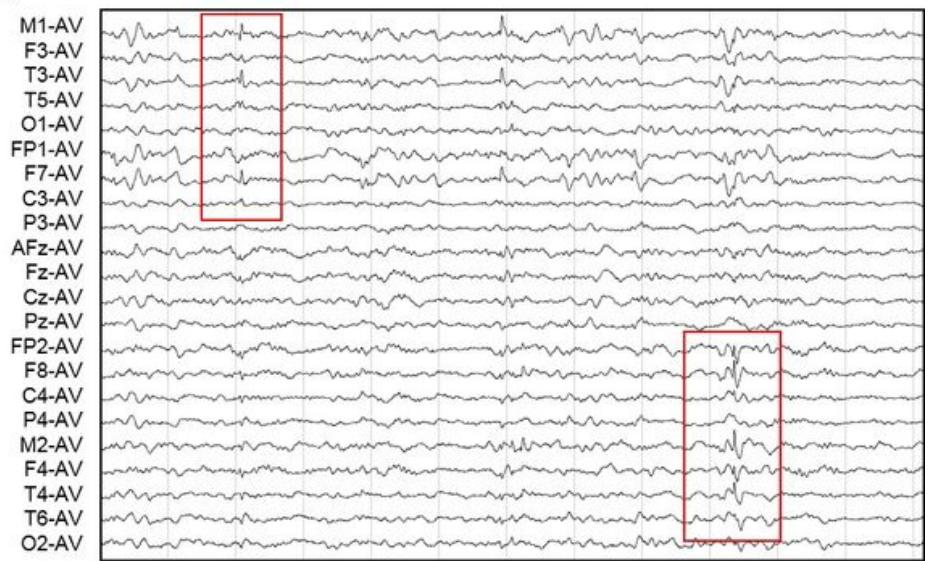

\section{Figure 1}

Some kinds of discharge patterns in interictal EEG (10-20 system). A. Observable interictal anterior discharges on the right (right temporal and frontal regions). B. Observable interictal multi-region discharges on the left. C. Noticeable interictal bilateral synchronous discharges. D. Noticeable interictal bilateral asynchronous discharges. 
A

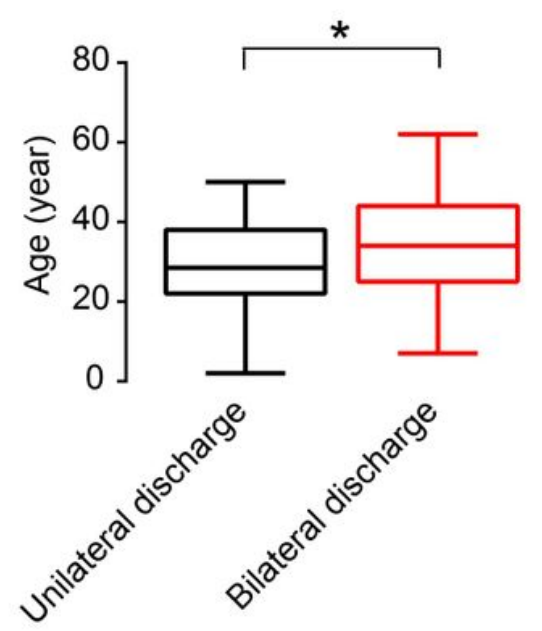

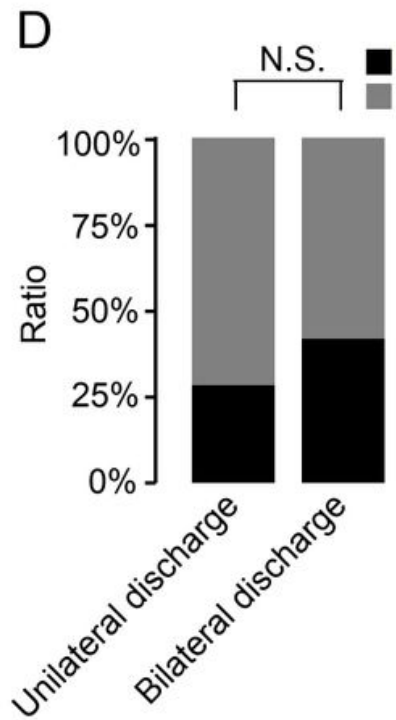

B

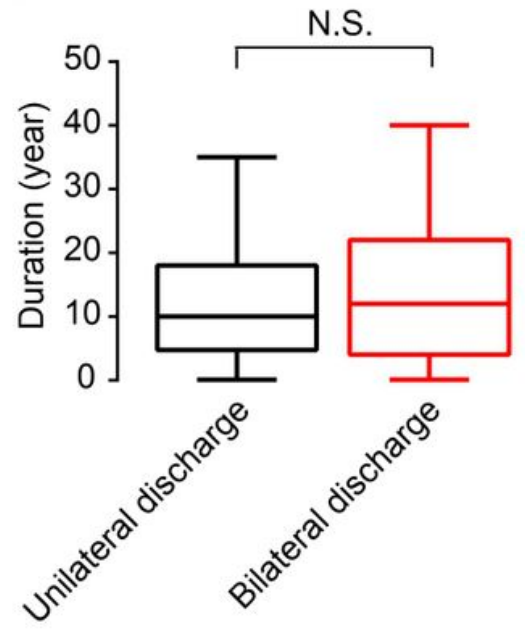

$E$
C

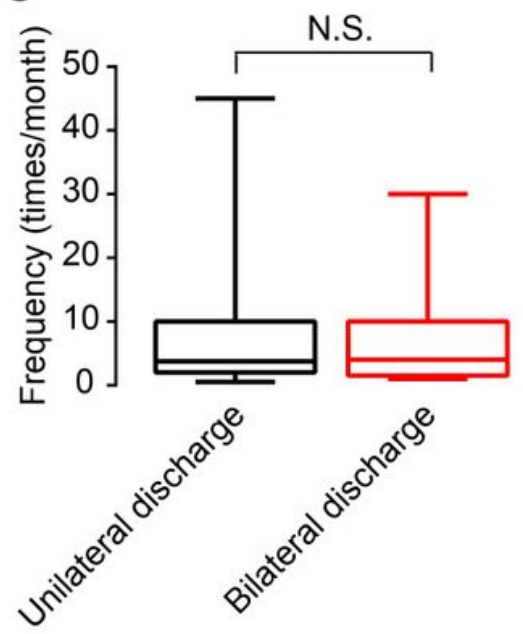

$\mathrm{F}$

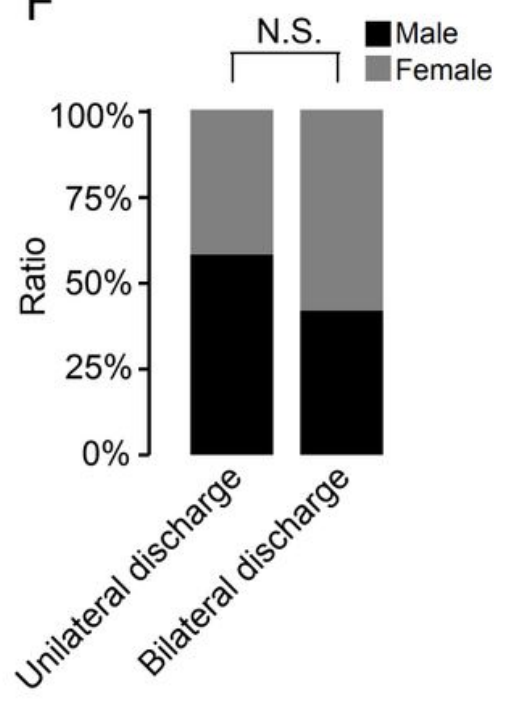

Figure 2

Effects of age, duration of disease, frequency of seizures, magnetic resonance imaging (MRI) findings, side of epilepsy, and gender on interictal discharge patterns in temporal lobe epilepsy (TLE). A. There was a statistically significant difference in age between the unilateral and bilateral discharge groups $(P<0.05)$; $B$. There was no statistically significant difference in the duration of disease between the two groups $(P>0.05)$; $C$. There was no statistically significant difference in seizure frequency between the two groups $(P>0.05)$; D. There was no statistically significant difference in the proportion of MRI manifestations between the two groups $(P>0.05)$; $E$. There was a statistically significant difference between the two groups in the proportion of right and left TLE $(P<0.05) ; F$. There was no statistically significant difference in the gender ratio between the two groups $(P>0.05)$. 


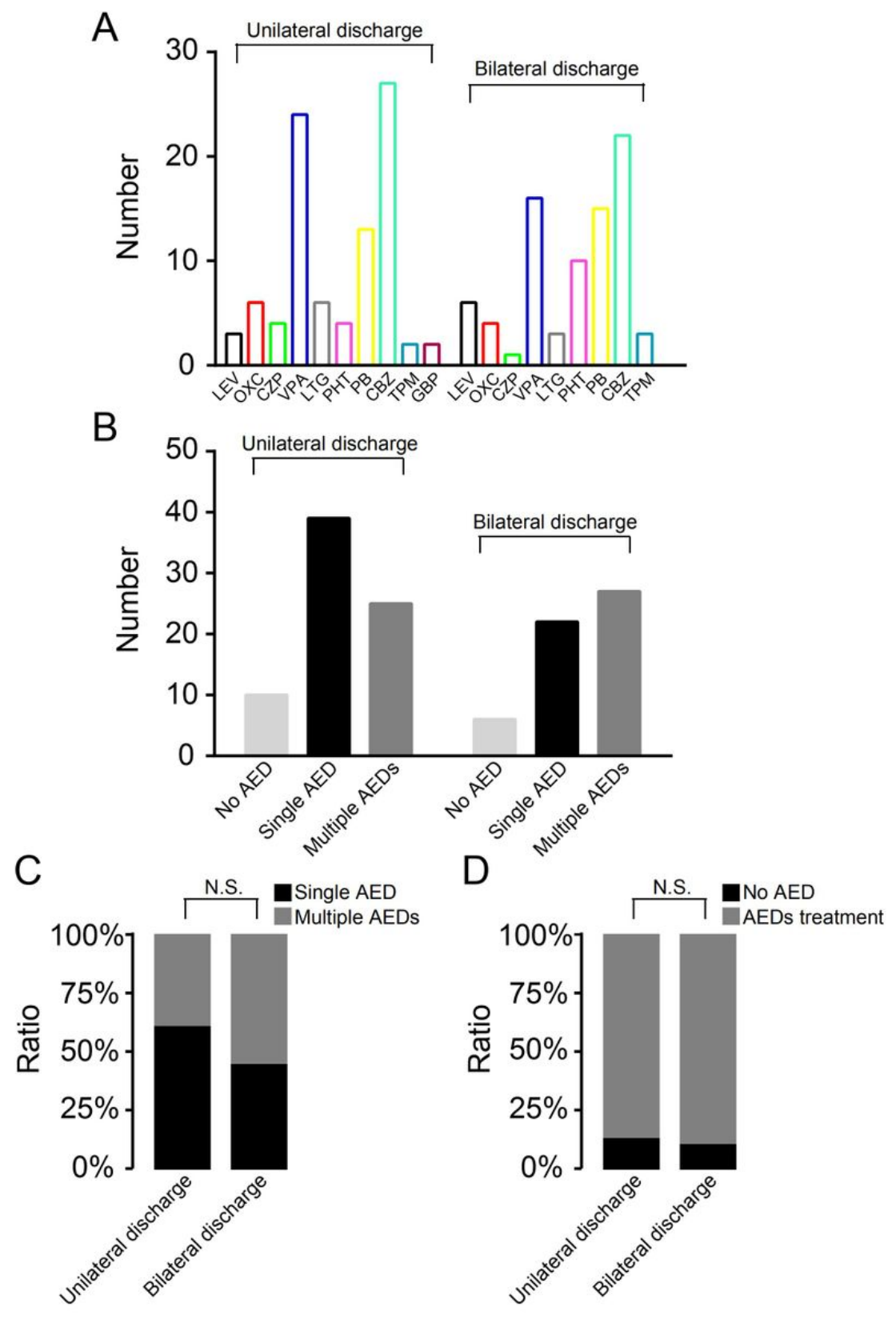

Figure 3

Administration of antiepileptic drugs (AEDs) and their effect on the interictal discharge pattern in temporal lobe epilepsy. A. Types of AEDs used in the unilateral and bilateral discharge groups; B. No AED administration, single AED administration, or multi-AED administration in the two groups; $C$. There was no statistically significant difference between the two groups in the proportion of single AED therapy patients and multi-AED therapy patients $(P>0.05)$; $D$. There was no statistically significant difference in the proportion of no AED therapy patients and single and multi-AED therapy patients between the two groups $(P>0.05)$. 
A

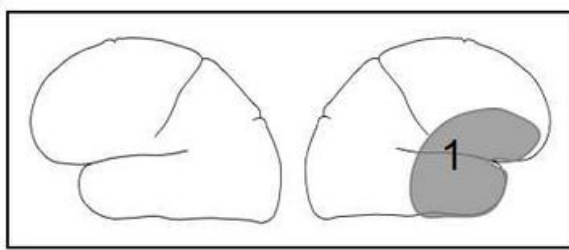

D

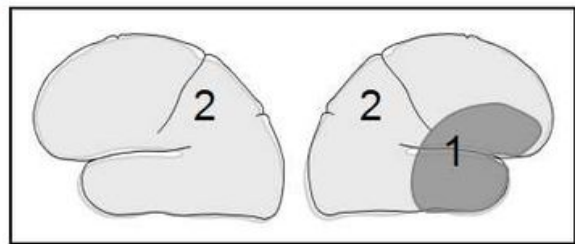

G

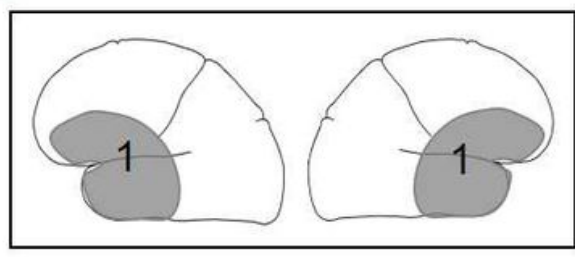

B

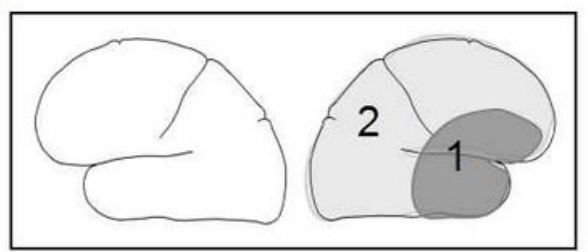

E

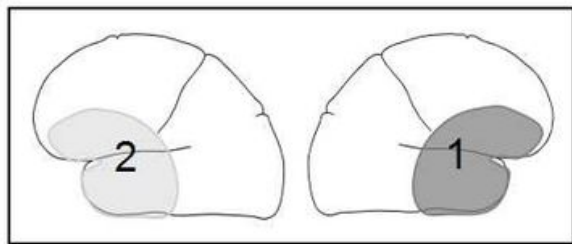

$\mathrm{H}$

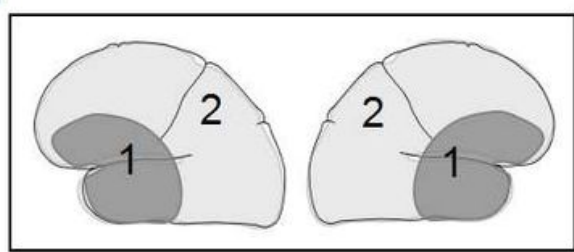

C

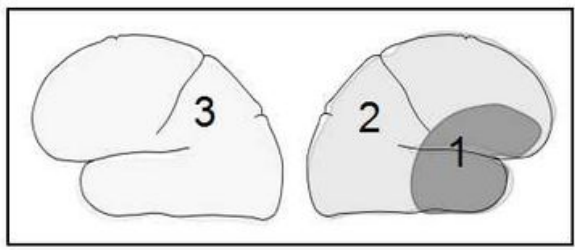

F

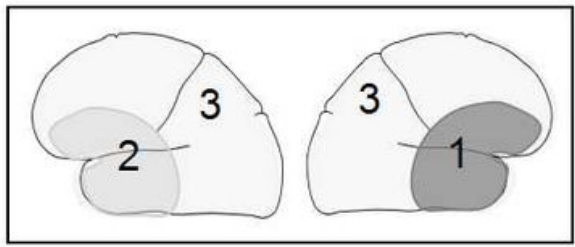

I

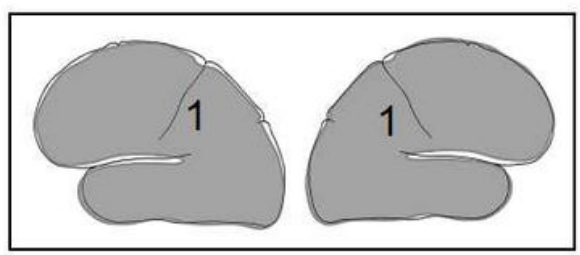

Figure 4

Ictal diffusion patterns in the right temporal lobe. A. Right anterior head; B. Right anterior head to right hemisphere; C. Right anterior head to right hemisphere and then to bilateral hemispheres; D. Right anterior head to bilateral hemispheres; E. Right anterior head to bilateral anterior heads; F. Right anterior head to bilateral anterior heads then to bilateral hemispheres; G. Bilateral anterior heads; $\mathrm{H}$. Bilateral anterior heads to bilateral hemispheres; I. Bilateral hemispheres. Note: The Numbers 1,2, and 3 represent diffusion order. 
A

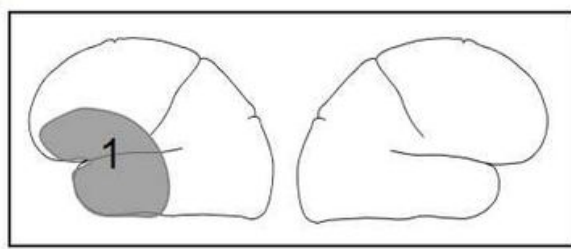

D

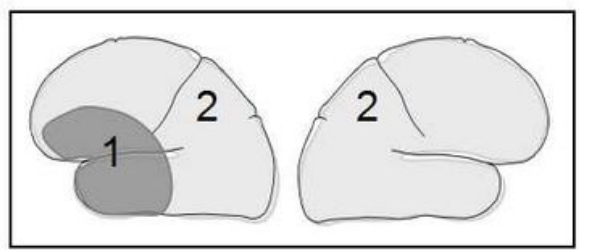

G

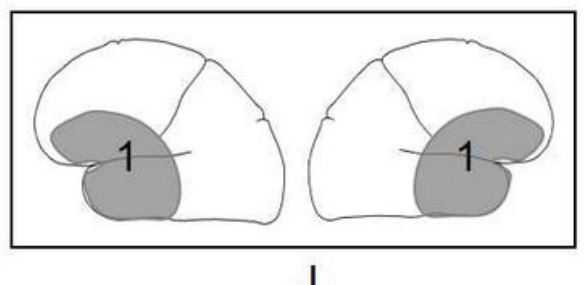

J

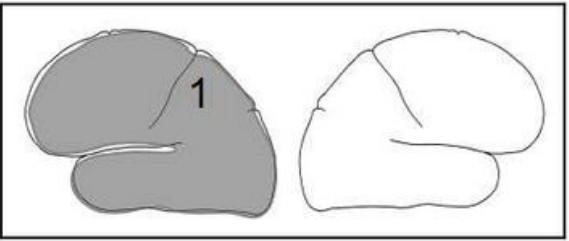

B

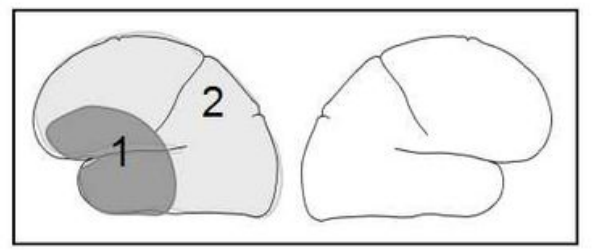

E

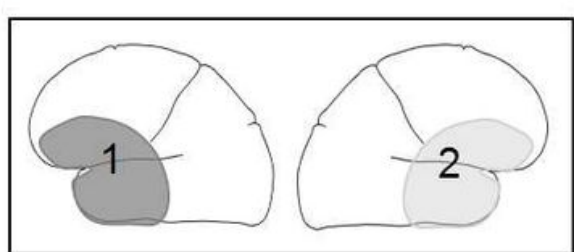

$\mathrm{H}$

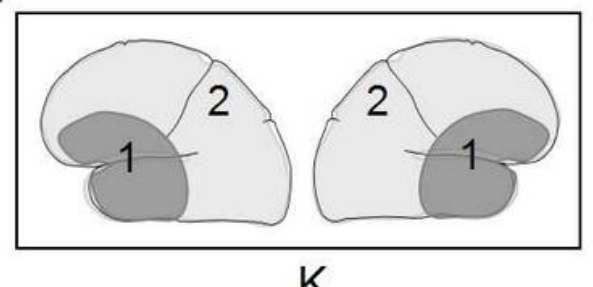

C

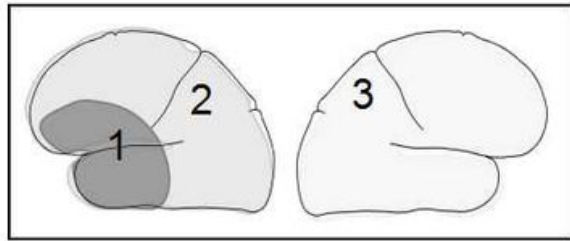

$\mathrm{F}$

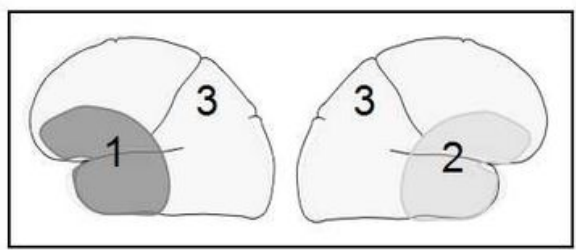

I

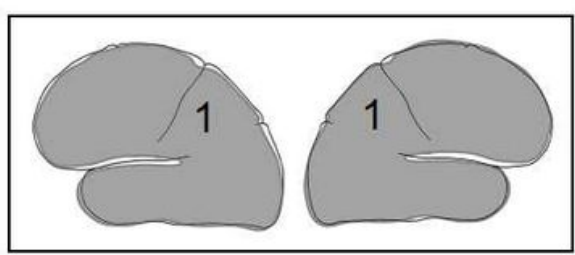

K

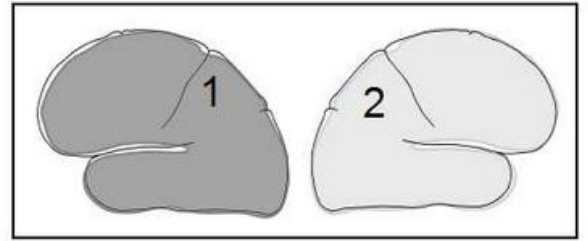

\section{Figure 5}

Ictal diffusion patterns in the left temporal lobe. A. Left anterior head; B. Left anterior head to left hemisphere; C. Left anterior head to left hemisphere and then to bilateral hemispheres; D. Left anterior head to bilateral hemispheres; E. Left anterior head to bilateral anterior heads; F. Left anterior head to bilateral anterior heads then to bilateral hemispheres; G. Bilateral anterior heads; $\mathrm{H}$. Bilateral anterior heads to bilateral hemispheres; I. Bilateral hemispheres; J. Left hemisphere; K. Left hemisphere to bilateral hemispheres. Note: The Numbers 1,2, and 3 represent diffusion order. 


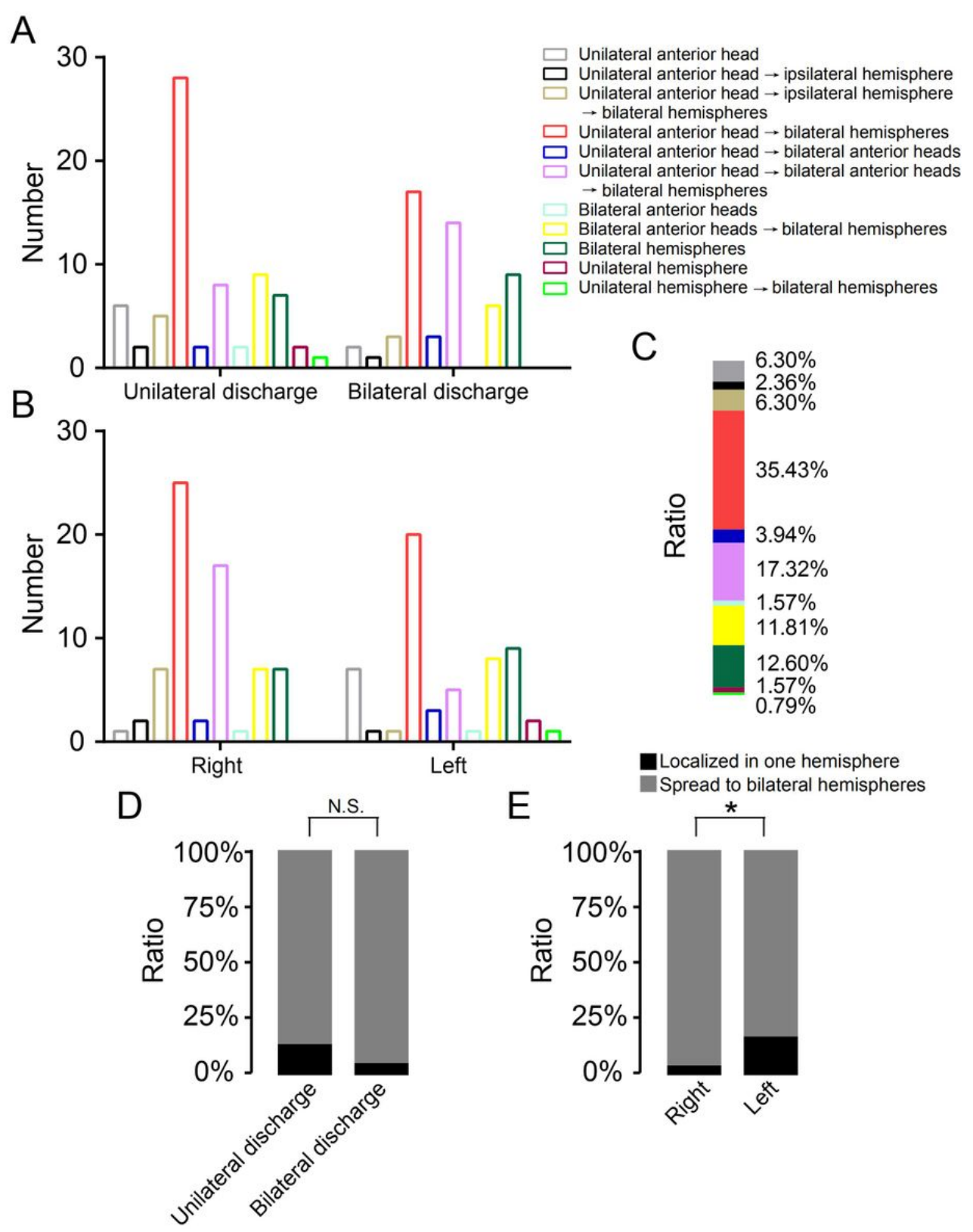

Figure 6

Patterns and differences in ictal diffusion in temporal lobe epilepsy (TLE). A. The ictal diffusion patterns in both unilateral and bilateral discharge groups; B. The ictal diffusion patterns in right and left TLE groups; C. Proportion of different ictal diffusion patterns in TLE; D. There was no statistically significant difference in the proportion of ictal EEG diffusion pattern confined to one hemisphere or diffused to bilateral hemispheres between the unilateral and bilateral discharge groups $(P>0.05)$; $E$. There was a statistically significant difference in the proportion of ictal EEG diffusion pattern confined to one hemisphere or diffused to bilateral hemispheres between the right and left TLE groups $(P<0.05)$. 